Journal of Advanced Research in Fluid Mechanics and Thermal Sciences

Journal homepage: www.akademiabaru.com/arfmts.html ISSN: 2289-7879

\title{
Analytical Solution of the Cauchy Problem for a Nonstationary Three- dimensional Model of the Filtration Theory
}

\author{
Aigerim T. Rakhymova1, ${ }^{*}$, Mars B. Gabbassov², Anvarjon A. Ahmedov ${ }^{3}$ \\ L.N.Gumilyov Eurasian National University, 010000 Nur Sultan, Kazakhstan \\ "Factor" System Research Company LLP, L.N.Gumilyov Eurasian National University, 010000 Nur Sultan, Kazakhstan \\ Pusat Sains Matematik, Universiti Malaysia Pahang, Lebuhraya Tun Razak, 26300 Gambang, Kuantan, Pahang, Malaysia
}

\section{ARTICLE INFO}

Article history:

Received 24 April 2021

Received in revised form 8 August 2021

Accepted 13 August 2021

Available online 10 September 2021

\section{Keywords:}

Four dimensional space; regular function; filtration; nonlinear equation; Cauchy problem

\section{ABSTRACT}

This paper is devoted to the study of the Cauchy problem for a system of differential equations describing the unsteady flow of a compressible fluid in a homogeneous and inhomogeneous porous medium with a general nonlinear filtration law in threedimensional space. In the work using the methods of four-dimensional mathematics, a special four-dimensional space was developed, as well as a functional space of regular functions, and analytical conditions were obtained on the general form of the nonlinear filtration law for which the Cauchy problem has a solution.

\section{Introduction}

A porous medium is understood as a material consisting of a solid matrix with an interconnected void. The interconnectedness of the voids allows one or more fluids to pass through the material. The simplest case is the motion of a single-phase liquid, where the pores are filled with one liquid. However, in natural cases, the pores are filled with liquids, gases, and mixtures of various types of liquids. Examples of natural porous media include soils, rocks, topsoil, sand, sandstone, limestone, etc. All these materials are capable to accumulate liquid in themselves and allow it to move under external forces $[1,4]$. In a natural porous environment, the distribution of pores in shape and size is uneven. At the pore scale (microscopic scale), the flow rate quantities (velocity, pressure, etc.) are clearly uneven, but in typical experiments the quantities are measured in areas that cross many pores, and such spatially averaged (macroscopic) quantities vary in a regular manner with respect to space and time, hence amenable to theoretical approach $[2,3]$.

Filtration is the movement of a fluid through a porous medium. The filtration law is the relationship between the filtration velocity and the pressure [5]. The study of the filtration process is a very interesting and economically important topic in connection with oil and gas production, the

\footnotetext{
* Corresponding author.

E-mail address: aigerim_rakhimova@mail.ru

https://doi.org/10.37934/arfmts.87.1.118133
} 
development of subsoil, where the main extraction technologies are leads by the laws of filtration theory.

Porosity is an important characteristic of porous media. The porosity coefficient $m$ is the ratio of the pore volume to the entire volume of the porous medium. The ability of a porous medium to pass liquid is characterized by permeability. The physical meaning of permeability is that it characterizes the cross-sectional area of the channels of a porous medium through which filtration occurs. The main filtration law is linear Darcy's law (in honor of the French engineer Henri Darcy, who experimentally established this law in 1856) [6-8]. Studies and experiments by G. Darcy on a steady unidirectional flow in a homogeneous medium revealed the proportionality between the flow rate and the pressure drop. It can be expressed as follows [1]

$u=-\frac{K}{\mu} \frac{\partial P}{\partial x}$

where $u$ is filtration velocity in $x$ direction, $\frac{\partial P}{\partial x}$ - pressure gradient in flow direction, $\mu$ - dynamic fluid viscosity, $K$ - specific or internal permeability of a porous medium. For the three-dimensional case, the Darcy equation can be expressed as follows

$\nabla P=-\frac{\mu}{K} \vec{v}$

where permeability $K$ is a second-order tensor (in the case of an isotropic medium, it is a scalar).

Darcy's law was confirmed by the results of many experiments, among which it is worth noting the authors of works, which provides a theoretical basis obtained in various ways using deterministic or statistical models $[9,10]$. Linear Darcy's law is valid (for both isotropic and anisotropic media) under the following conditions, that the filtration rate and the pressure gradient is small and the change in filtration rate and pressure gradient also small. However, a huge amount of research has shown that there are limits to the applicability of linear Darcy's law. The upper limit is determined in the presence of inertial forces at high filtration rates, i.e., the upper limit of applicability of Darcy's law is determined by the critical Reynolds number $1<R e_{c r}<12$ [11]. A lot of researches have been devoted to the study of fluid motion taking into account inertial effects in a porous medium. Among them, it is worth noting the works, where restrictions on the use of the linear Darcy's law are considered and the nonlinear laws of fluid motion are studied in the presence of a Reynolds number greater than $R e_{c r}$ [12-14]. The lower limit of the linear Darcy's law applicability is determined by the existing of non-Newtonian rheological properties of the liquid, its interaction with the solid skeleton of a porous medium at sufficiently low filtration rates.

As mentioned above, at the Reynolds number $R e>R e_{c r}$ the linear Darcy's law ceases to be valid. The first generalization of Darcy's law to the case of large $R e$ was founded by Dupuis, who formulated a two-term filtration law. Later, the Austrian researcher F. Forchheimer independently established two-term filtration law, which is still called F. Forchheimer equation, and its generalization with a nonstationary effect was proposed by the authors of papers $[7,12,13,15,16]$. A lot of scientific papers are devoted to the numerical solution of liquid flow problems $[17,18]$.

The solution of nonlinear filtration problems is of great interest $[19,20]$. Among them, note the investigation of B.V. Alekseev, who proposed an analytical solution to the nonlinear Leibenson equation, which is the problem of transient filtration of a perfect gas.

The aim of this research is to obtain an analytical solution of three-dimensional filtration problem. In an attempt to obtain an analytical solution of a three-dimensional mathematical model, we 
propose a new approach to solving the problem in the space of four-dimensional numbers, first proposed by the Kazakh mathematician Abenov [21] (Kazakh National University named after alFarabi). Abenov and Gabbassov [23] described anisotropic four-dimensional spaces M2-M7, which are associative and commutative with zero divisors. In what follows, these spaces will be called Abenov spaces. In this paper, we propose an analytical solution to a mathematical filtration model with a nonlinear Darcy's law in the four-dimensional Abenov space M5.

Mainly the three-dimensional problems of fluid motion are mainly solved by numerical methods, obtaining an analytical solution of them is difficult. To avoid this difficulty, Abenov [21] and Abenov et al., [22] proposed a new method for solving the continuity equation in four-dimensional space. Rakhymova et al., [24] presented elements of the Abenov space M5. In particular, if a function $U\left(x_{1}, x_{2}, x_{3}, x_{4}\right)=\left(u_{1}, u_{2}, u_{3}, u_{4}\right)$ has a derivative, then it must satisfy the Cauchy-Riemann condition [24]

$$
\left\{\begin{array}{c}
\frac{\partial u_{1}}{\partial x_{1}}=\frac{\partial u_{2}}{\partial x_{2}}=\frac{\partial u_{3}}{\partial x_{3}}=\frac{\partial u_{4}}{\partial x_{4}} \\
\frac{\partial u_{2}}{\partial x_{1}}=-\frac{\partial u_{1}}{\partial x_{2}}=\frac{\partial u_{4}}{\partial x_{3}}=-\frac{\partial u_{3}}{\partial x_{4}} \\
\frac{\partial u_{3}}{\partial x_{1}}=\frac{\partial u_{4}}{\partial x_{2}}=-\frac{\partial u_{1}}{\partial x_{3}}=-\frac{\partial u_{2}}{\partial x_{4}} \\
\frac{\partial u_{4}}{\partial x_{1}}=-\frac{\partial u_{3}}{\partial x_{2}}=-\frac{\partial u_{2}}{\partial x_{3}}=\frac{\partial u_{1}}{\partial x_{4}}
\end{array} .\right.
$$

\section{Methodology}

Consider an equation with four unknowns of the following form

$\operatorname{div} U=\frac{\partial u_{1}}{\partial x_{1}}+\frac{\partial u_{2}}{\partial x_{2}}+\frac{\partial u_{3}}{\partial x_{3}}+\frac{\partial u_{4}}{\partial x_{4}}=0$

Definition: Four-dimensional function $U\left(x_{1}, x_{2}, x_{3}, x_{4}\right)=\left(u_{1}, u_{2}, u_{3}, u_{4}\right)$, which is a solution to Eq. (1) is called the solenoidal field.

Theorem 1: One class of general solutions of Eq. (1) in the space M5 has the form

$$
\left\{\begin{array}{l}
u_{1}=q_{1} w_{1}\left(p_{1} x_{1}, p_{2} x_{2}, p_{3} x_{3}, p_{4} x_{4}\right) \\
u_{2}=q_{2} w_{2}\left(p_{1} x_{1}, p_{2} x_{2}, p_{3} x_{3}, p_{4} x_{4}\right) \\
u_{3}=q_{3} w_{3}\left(p_{1} x_{1}, p_{2} x_{2}, p_{3} x_{3}, p_{4} x_{4}\right)^{\prime} \\
u_{4}=q_{4} w_{4}\left(p_{1} x_{1}, p_{2} x_{2}, p_{3} x_{3}, p_{4} x_{4}\right)
\end{array}\right.
$$

where $W(X)=W\left(x_{1}, x_{2}, x_{3}, x_{4}\right)=\left(w_{1}, w_{2}, w_{3}, w_{4}\right)$ - any regular function, $p_{i}, q_{i},(i=1,2,3,4)-$ random real constants satisfying the relation

$\sum_{i=1}^{4} p_{i} q_{i}=0$.

Proof: Substituting function (2) into Eq. (1), we obtain 
$\operatorname{div} U=p_{1} q_{1} \frac{\partial w_{1}\left(p_{1} x_{1}, p_{2} x_{2}, p_{3} x_{3}, p_{4} x_{4}\right)}{\partial x_{1}}+p_{2} q_{2} \frac{\partial w_{2}\left(p_{1} x_{1}, p_{2} x_{2}, p_{3} x_{3}, p_{4} x_{4}\right)}{\partial y_{2}}+p_{3} q_{3} \frac{\partial w_{3}\left(p_{1} x_{1}, p_{2} x_{2}, p_{3} x_{3}, p_{4} x_{4}\right)}{\partial y_{3}}+$ $p_{4} q_{4} \frac{\partial w_{4}\left(p_{1} x_{1}, p_{2} x_{2}, p_{3} x_{3}, p_{4} x_{4}\right)}{\partial y_{4}}=\sum_{i=1}^{4} p_{i} q_{i} \cdot \frac{\partial w_{1}\left(p_{1} x_{1}, p_{2} x_{2}, p_{3} x_{3}, p_{4} x_{4}\right)}{\partial x_{1}}=0$,

by (3) and the Cauchy-Riemann conditions.

The theorem is proved.

An interesting question is the possibility of uniquely recovering the solenoidal field according to additional conditions, that is, under what additional conditions on the function $U\left(x_{1}, x_{2}, x_{3}, x_{4}\right)=$ $\left(u_{1}, u_{2}, u_{3}, u_{4}\right)$ is it possible to uniquely determine the solution of Eq. (1) from one of spaces $S_{5}^{1}$. Let us set the initial Cauchy data as additional conditions. Suppose that at $x_{4}=0$ for the solution of Eq.

(1) the following initial conditions are given

$u_{1}\left(x_{1}, x_{2}, x_{3}, 0\right)=u_{10}\left(x_{1}, x_{2}, x_{3}\right)$
$u_{2}\left(x_{1}, x_{2}, x_{3}, 0\right)=u_{20}\left(x_{1}, x_{2}, x_{3}\right)$
$u_{3}\left(x_{1}, x_{2}, x_{3}, 0\right)=u_{30}\left(x_{1}, x_{2}, x_{3}\right)$
$u_{4}\left(x_{1}, x_{2}, x_{3}, 0\right)=u_{40}\left(x_{1}, x_{2}, x_{3}\right)$

Theorem 2: Let the functions $u_{10}, u_{20}, u_{30}, u_{40}$ defining the initial conditions (5) belong to the class $C^{1}\left(R^{3}\right)$ and satisfy the conditions

$$
\left\{\begin{array}{c}
\frac{\partial u_{10}}{\partial x_{1}}=s_{12} \frac{\partial u_{20}}{\partial x_{2}}=s_{13} \frac{\partial u_{30}}{\partial x_{3}} \\
\frac{\partial u_{20}}{\partial x_{1}}=-s_{21} \frac{\partial u_{10}}{\partial x_{2}}=s_{13} \frac{\partial u_{40}}{\partial x_{3}} \\
\frac{\partial u_{30}}{\partial x_{1}}=s_{12} \frac{\partial u_{40}}{\partial x_{2}}=-s_{31} \frac{\partial u_{10}}{\partial x_{3}} \\
\frac{\partial u_{40}}{\partial x_{1}}=-s_{21} \frac{\partial u_{30}}{\partial x_{2}}=-s_{31} \frac{\partial u_{20}}{\partial x_{3}}
\end{array}\right.
$$

for $\left(x_{1}, x_{2}, x_{3}\right) \in R^{3}$, where $s_{12}, s_{21}, s_{13}, s_{31}$ given positive real constants. Then the Cauchy problem (1), (5) has a unique solution from the class $S_{5}^{1}\left(R^{4}\right)$ and it is expressed by the formulas

$$
\begin{aligned}
& u_{1}\left(x_{1}, x_{2}, x_{3}, x_{4}\right)=\frac{1}{2}\left[u_{10}\left(x_{1}+\frac{s_{12} s_{13}+s_{12}+s_{13}}{\sqrt{s_{12} s_{21} s_{13} s_{31}}} x_{4}, x_{2}, x_{3}\right)+u_{10}\left(x_{1}-\frac{s_{12} s_{13}+s_{12}+s_{13}}{\sqrt{s_{12} s_{21} s_{13} s_{31}}} x_{4}, x_{2}, x_{3}\right)+\right. \\
& \left.\sqrt{\frac{s_{12} s_{13}}{s_{21} s_{31}}}\left(u_{40}\left(x_{1}+\frac{s_{12} s_{13}+s_{12}+s_{13}}{\sqrt{s_{12} s_{21} s_{13} s_{31}}} x_{4}, x_{2}, x_{3}\right)-u_{40}\left(x_{1}-\frac{s_{12} s_{13}+s_{12}+s_{13}}{\sqrt{s_{12} s_{21} s_{13} s_{31}}} x_{4}, x_{2}, x_{3}\right)\right)\right], \\
& u_{2}\left(x_{1}, x_{2}, x_{3}, x_{4}\right)=\frac{1}{2}\left[u_{20}\left(x_{1}+\frac{s_{12} s_{13}+s_{12}+s_{13}}{\sqrt{s_{12} s_{21} s_{13} s_{31}}} x_{4}, x_{2}, x_{3}\right)+u_{20}\left(x_{1}-\frac{s_{12} s_{13}+s_{12}+s_{13}}{\sqrt{s_{12} s_{21} s_{13} s_{31}}} x_{4}, x_{2}, x_{3}\right)-\right. \\
& \left.\sqrt{\frac{s_{21} s_{13}}{s_{12} s_{31}}}\left(u_{30}\left(x_{1}+\frac{s_{12} s_{13}+s_{12}+s_{13}}{\sqrt{s_{12} s_{21} s_{13} s_{31}}} x_{4}, x_{2}, x_{3}\right)-u_{30}\left(x_{1}-\frac{s_{12} s_{13}+s_{12}+s_{13}}{\sqrt{s_{12} s_{21} s_{13} s_{31}}} x_{4}, x_{2}, x_{3}\right)\right)\right], \\
& u_{3}\left(x_{1}, x_{2}, x_{3}, x_{4}\right)=\frac{1}{2}\left[u_{30}\left(x_{1}+\frac{s_{12} s_{13}+s_{12}+s_{13}}{\sqrt{s_{12} s_{21} s_{13} s_{31}}} x_{4}, x_{2}, x_{3}\right)+u_{30}\left(x_{1}-\frac{s_{12} s_{13}+s_{12}+s_{13}}{\sqrt{s_{12} s_{21} s_{13} s_{31}}} x_{4}, x_{2}, x_{3}\right)-\right. \\
& \left.\sqrt{\frac{s_{12} s_{31}}{s_{21} s_{13}}}\left(u_{20}\left(x_{1}+\frac{s_{12} s_{13}+s_{12}+s_{13}}{\sqrt{s_{12} s_{21} s_{13} s_{31}}} x_{4}, x_{2}, x_{3}\right)-u_{20}\left(x_{1}-\frac{s_{12} s_{13}+s_{12}+s_{13}}{\sqrt{s_{12} s_{21} s_{13} s_{31}}} x_{4}, x_{2}, x_{3}\right)\right)\right],
\end{aligned}
$$




$$
\begin{aligned}
& u_{4}\left(x_{1}, x_{2}, x_{3}, x_{4}\right)=\frac{1}{2}\left[u_{40}\left(x_{1}+\frac{s_{12} s_{13}+s_{12}+s_{13}}{\sqrt{s_{12} s_{21} s_{13} s_{31}}} x_{4}, x_{2}, x_{3}\right)+u_{40}\left(x_{1}-\frac{s_{12} s_{13}+s_{12}+s_{13}}{\sqrt{s_{12} s_{21} s_{13} s_{31}}} x_{4}, x_{2}, x_{3}\right)+\right. \\
& \left.\sqrt{\frac{s_{12} s_{13}}{s_{21} s_{31}}}\left(u_{10}\left(x_{1}-\frac{s_{12} s_{13}+s_{12}+s_{13}}{\sqrt{s_{12} s_{21} s_{13} s_{31}}} x_{4}, x_{2}, x_{3}\right)-u_{10}\left(x_{1}-\frac{s_{12} s_{13}+s_{12}+s_{13}}{\sqrt{s_{12} s_{21} s_{13} s_{31}}} x_{4}, x_{2}, x_{3}\right)\right)\right] .
\end{aligned}
$$

Proof: We find a solution to problem (1), (5) in the form (2) with condition (3). Then from the Cauchy-Riemann conditions for the function $W(X)=W\left(x_{1}, x_{2}, x_{3}, x_{4}\right)=\left(w_{1}, w_{2}, w_{3}, w_{4}\right)$, it follows that the function $U(X)=U\left(x_{1}, x_{2}, x_{3}, x_{4}\right)=\left(u_{1}, u_{2}, u_{3}, u_{4}\right)$ satisfy the following conditions

$$
\begin{aligned}
& \frac{1}{p_{1} q_{1}} \frac{\partial u_{1}}{\partial x_{1}}=\frac{1}{p_{2} q_{2}} \frac{\partial u_{2}}{\partial x_{2}}=\frac{1}{p_{3} q_{3}} \frac{\partial u_{3}}{\partial x_{3}}=\frac{1}{p_{4} q_{4}} \frac{\partial u_{4}}{\partial x_{4}} \\
& \frac{1}{p_{1} q_{2}} \frac{\partial u_{2}}{\partial x_{1}}=-\frac{1}{p_{2} q_{1}} \frac{\partial u_{1}}{\partial x_{2}}=\frac{1}{p_{3} q_{4}} \frac{\partial u_{4}}{\partial x_{3}}=-\frac{1}{p_{4} q_{3}} \frac{\partial u_{3}}{\partial x_{4}} \\
& \frac{1}{p_{1} q_{3}} \frac{\partial u_{3}}{\partial x_{1}}=\frac{1}{p_{2} q_{4}} \frac{\partial u_{4}}{\partial x_{2}}=-\frac{1}{p_{3} q_{1}} \frac{\partial u_{1}}{\partial x_{3}}=-\frac{1}{p_{4} q_{2}} \frac{\partial u_{2}}{\partial x_{4}} \\
& \frac{1}{p_{1} q_{4}} \frac{\partial u_{4}}{\partial x_{1}}=-\frac{1}{p_{2} q_{3}} \frac{\partial u_{3}}{\partial x_{2}}=-\frac{1}{p_{3} q_{2}} \frac{\partial u_{2}}{\partial x_{3}}=\frac{1}{p_{4} q_{1}} \frac{\partial u_{1}}{\partial x_{4}}
\end{aligned}
$$

From these equations it is easy to obtain that each function $u_{i}\left(x_{1}, x_{2}, x_{3}, x_{4}\right), i=1,2,3,4$, satisfies the following equations

$$
\begin{aligned}
& \frac{\partial^{2} u_{i}}{\partial x_{4}^{2}}=\frac{p_{4}^{2}}{p_{1}^{2}} \frac{\partial^{2} u_{i}}{\partial x_{1}^{2}} \\
& \frac{\partial^{2} u_{i}}{\partial x_{4}^{2}}=-\frac{p_{4}^{2}}{p_{2}^{2}} \frac{\partial^{2} u_{i}}{\partial x_{2}^{2}}, \\
& \frac{\partial^{2} u_{i}}{\partial x_{4}^{2}}=-\frac{p_{4}^{2}}{p_{3}^{2}} \frac{\partial^{2} u_{i}}{\partial x_{3}^{2}},
\end{aligned}
$$

as well as additional initial conditions, except for (5)

$$
\begin{aligned}
& \frac{\partial u_{1}\left(x_{1}, x_{2}, x_{3}, 0\right)}{\partial x_{4}}=\frac{p_{4} q_{1}}{p_{1} q_{4}} \frac{\partial u_{40}\left(x_{1}, x_{2}, x_{3}\right)}{\partial x_{1}}=-\frac{p_{4} q_{1}}{p_{2} q_{3}} \frac{\partial u_{30}\left(x_{1}, x_{2}, x_{3}\right)}{\partial x_{2}}=-\frac{p_{4} q_{1}}{p_{3} q_{2}} \frac{\partial u_{20}\left(x_{1}, x_{2}, x_{3}\right)}{\partial x_{3}}, \\
& \frac{\partial u_{2}\left(x_{1}, x_{2}, x_{3}, 0\right)}{\partial x_{4}}=-\frac{p_{4} q_{2}}{p_{1} q_{3}} \frac{\partial u_{30}\left(x_{1}, x_{2}, x_{3}\right)}{\partial x_{1}}=-\frac{p_{4} q_{2}}{p_{2} q_{4}} \frac{\partial u_{40}\left(x_{1}, x_{2}, x_{3}\right)}{\partial x_{2}}=\frac{p_{4} q_{2}}{p_{3} q_{1}} \frac{\partial u_{10}\left(x_{1}, x_{2}, x_{3}\right)}{\partial x_{3}}, \\
& \frac{\partial u_{3}\left(x_{1}, x_{2}, x_{3}, 0\right)}{\partial x_{4}}=-\frac{p_{4} q_{3}}{p_{1} q_{2}} \frac{\partial u_{20}\left(x_{1}, x_{2}, x_{3}\right)}{\partial x_{1}}=\frac{p_{4} q_{3}}{p_{2} q_{1}} \frac{\partial u_{10}\left(x_{1}, x_{2}, x_{3}\right)}{\partial x_{2}}=-\frac{p_{4} q_{3}}{p_{3} q_{4}} \frac{\partial u_{40}\left(x_{1}, x_{2}, x_{3}\right)}{\partial x_{3}}, \\
& \frac{\partial u_{4}\left(x_{1}, x_{2}, x_{3}, 0\right)}{\partial x_{4}}=\frac{p_{4} q_{4}}{p_{1} q_{1}} \frac{\partial u_{10}\left(x_{1}, x_{2}, x_{3}\right)}{\partial x_{1}}=\frac{p_{4} q_{4}}{p_{2} q_{2}} \frac{\partial u_{20}\left(x_{1}, x_{2}, x_{3}\right)}{\partial x_{2}}=\frac{p_{4} q_{4}}{p_{3} q_{3}} \frac{\partial u_{30}\left(x_{1}, x_{2}, x_{3}\right)}{\partial x_{3}} .
\end{aligned}
$$

From these conditions it follows that the functions $u_{10}, u_{20}, u_{30}, u_{40}$ setting the initial conditions (5) must satisfy the equations

$$
\begin{aligned}
& \frac{p_{4} q_{1}}{p_{1} q_{4}} \frac{\partial u_{40}\left(x_{1}, x_{2}, x_{3}\right)}{\partial x_{1}}=-\frac{p_{4} q_{1}}{p_{2} q_{3}} \frac{\partial u_{30}\left(x_{1}, x_{2}, x_{3}\right)}{\partial x_{2}}=-\frac{p_{4} q_{1}}{p_{3} q_{2}} \frac{\partial u_{20}\left(x_{1}, x_{2}, x_{3}\right)}{\partial x_{3}}, \\
& -\frac{p_{4} q_{2}}{p_{1} q_{3}} \frac{\partial u_{30}\left(x_{1}, x_{2}, x_{3}\right)}{\partial x_{1}}=-\frac{p_{4} q_{2}}{p_{2} q_{4}} \frac{\partial u_{40}\left(x_{1}, x_{2}, x_{3}\right)}{\partial x_{2}}=\frac{p_{4} q_{2}}{p_{3} q_{1}} \frac{\partial u_{10}\left(x_{1}, x_{2}, x_{3}\right)}{\partial x_{3}}, \\
& -\frac{p_{4} q_{3}}{p_{1} q_{2}} \frac{\partial u_{20}\left(x_{1}, x_{2}, x_{3}\right)}{\partial x_{1}}=\frac{p_{4} q_{3}}{p_{2} q_{1}} \frac{\partial u_{10}\left(x_{1}, x_{2}, x_{3}\right)}{\partial x_{2}}=-\frac{p_{4} q_{3}}{p_{3} q_{4}} \frac{\partial u_{40}\left(x_{1}, x_{2}, x_{3}\right)}{\partial x_{3}},
\end{aligned}
$$


$\frac{p_{4} q_{4}}{p_{1} q_{1}} \frac{\partial u_{10}\left(x_{1}, x_{2}, x_{3}\right)}{\partial x_{1}}=\frac{p_{4} q_{4}}{p_{2} q_{2}} \frac{\partial u_{20}\left(x_{1}, x_{2}, x_{3}\right)}{\partial x_{2}}=\frac{p_{4} q_{4}}{p_{3} q_{3}} \frac{\partial u_{30}\left(x_{1}, x_{2}, x_{3}\right)}{\partial x_{3}}$.

Thus, the function $u_{1}\left(x_{1}, x_{2}, x_{3}, x_{4}\right)$ is a solution to the following Cauchy problem

$\frac{\partial^{2} u_{1}\left(x_{1}, x_{2}, x_{3}, x_{4}\right)}{\partial x_{4}^{2}}=a^{2} \frac{\partial^{2} u_{1}\left(x_{1}, x_{2}, x_{3}, x_{4}\right)}{\partial x_{1}^{2}}$,

$u_{1}\left(x_{1}, x_{2}, x_{3}, 0\right)=u_{10}\left(x_{1}, x_{2}, x_{3}\right)$,

$\frac{\partial u_{1}\left(x_{1}, x_{2}, x_{3}, 0\right)}{\partial x_{4}}=\frac{p_{4} q_{1}}{p_{1} q_{4}} \frac{\partial u_{40}\left(x_{1}, x_{2}, x_{3}\right)}{\partial x_{1}}$

where $a=\frac{p_{4}}{p_{1}}$. According to the d'Alembert formula, the solution to this problem has the form [21]

$u_{1}\left(x_{1}, x_{2}, x_{3}, x_{4}\right)=\frac{1}{2}\left(u_{10}\left(x_{1}+a x_{4}, x_{2}, x_{3}\right)+u_{10}\left(x_{1}-a x_{4}, x_{2}, x_{3}\right)\right)+\frac{1}{2} \frac{q_{1}}{q_{4}}\left(u_{40}\left(x_{1}+\right.\right.$

$\left.\left.a x_{4}, x_{2}, x_{3}\right)-u_{40}\left(x_{1}-a x_{4}, x_{2}, x_{3}\right)\right), x_{4}>0,\left(x_{1}, x_{2}, x_{3}\right) \in R^{3}$.

Similarly, the function $u_{2}\left(x_{1}, x_{2}, x_{3}, x_{4}\right)$ is a solution to the following Cauchy problem:

$\frac{\partial^{2} u_{2}\left(x_{1}, x_{2}, x_{3}, x_{4}\right)}{\partial x_{4}^{2}}=a^{2} \frac{\partial^{2} u_{2}\left(x_{1}, x_{2}, x_{3}, x_{4}\right)}{\partial x_{1}^{2}}$

$u_{2}\left(x_{1}, x_{2}, x_{3}, 0\right)=u_{20}\left(x_{1}, x_{2}, x_{3}\right)$,

$\frac{\partial u_{2}\left(x_{1}, x_{2}, x_{3}, 0\right)}{\partial x_{4}}=-\frac{p_{4} q_{2}}{p_{1} q_{3}} \frac{\partial u_{30}\left(x_{1}, x_{2}, x_{3}\right)}{\partial x_{1}}$.

The solution to this problem is

$u_{2}\left(x_{1}, x_{2}, x_{3}, x_{4}\right)=\frac{1}{2}\left(u_{20}\left(x_{1}+a x_{4}, x_{2}, x_{3}\right)+u_{20}\left(x_{1}-a x_{4}, x_{2}, x_{3}\right)\right)-\frac{1}{2} \frac{q_{2}}{q_{3}}\left(u_{30}\left(x_{1}+\right.\right.$

$\left.\left.a x_{4}, x_{2}, x_{3}\right)-u_{30}\left(x_{1}-a x_{4}, x_{2}, x_{3}\right)\right), x_{4}>0,\left(x_{1}, x_{2}, x_{3}\right) \in R^{3}$.

The function $u_{3}\left(x_{1}, x_{2}, x_{3}, x_{4}\right)$ is a solution to the Cauchy problem

$\frac{\partial^{2} u_{3}\left(x_{1}, x_{2}, x_{3}, x_{4}\right)}{\partial x_{4}^{2}}=a^{2} \frac{\partial^{2} u_{3}\left(x_{1}, x_{2}, x_{3}, x_{4}\right)}{\partial x_{1}^{2}}$,

$u_{3}\left(x_{1}, x_{2}, x_{3}, 0\right)=u_{30}\left(x_{1}, x_{2}, x_{3}\right)$,

$\frac{\partial u_{3}\left(x_{1}, x_{2}, x_{3}, 0\right)}{\partial x_{4}}=-\frac{p_{4} q_{3}}{p_{1} q_{2}} \frac{\partial u_{20}\left(x_{1}, x_{2}, x_{3}\right)}{\partial x_{1}}$.

Then,

$u_{3}\left(x_{1}, x_{2}, x_{3}, x_{4}\right)=\frac{1}{2}\left(u_{30}\left(x_{1}+a x_{4}, x_{2}, x_{3}\right)+u_{30}\left(x_{1}-a x_{4}, x_{2}, x_{3}\right)\right)-\frac{1}{2} \frac{q_{3}}{q_{2}}\left(u_{20}\left(x_{1}+\right.\right.$

$\left.\left.a x_{4}, x_{2}, x_{3}\right)-u_{20}\left(x_{1}-a x_{4}, x_{2}, x_{3}\right)\right), x_{4}>0,\left(x_{1}, x_{2}, x_{3}\right) \in R^{3}$.

The function $u_{4}\left(x_{1}, x_{2}, x_{3}, x_{4}\right)$ is a solution to the Cauchy problem

$$
\begin{aligned}
& \frac{\partial^{2} u_{4}\left(x_{1}, x_{2}, x_{3}, x_{4}\right)}{\partial x_{4}^{2}}=a^{2} \frac{\partial^{2} u_{4}\left(x_{1}, x_{2}, x_{3}, x_{4}\right)}{\partial x_{1}^{2}}, \\
& u_{4}\left(x_{1}, x_{2}, x_{3}, 0\right)=u_{40}\left(x_{1}, x_{2}, x_{3}\right) \\
& \frac{\partial u_{4}\left(x_{1}, x_{2}, x_{3}, 0\right)}{\partial x_{4}}=\frac{p_{4} q_{4}}{p_{1} q_{1}} \frac{\partial u_{10}\left(x_{1}, x_{2}, x_{3}\right)}{\partial x_{1}} .
\end{aligned}
$$


Then,

$$
\begin{aligned}
& u_{4}\left(x_{1}, x_{2}, x_{3}, x_{4}\right)=\frac{1}{2}\left(u_{40}\left(x_{1}+a x_{4}, x_{2}, x_{3}\right)+u_{40}\left(x_{1}-a x_{4}, x_{2}, x_{3}\right)\right)+\frac{1}{2} \frac{q_{4}}{q_{1}}\left(u _ { 1 0 } \left(x_{1}+\right.\right. \\
& \left.\left.a x_{4}, x_{2}, x_{3}\right)-u_{10}\left(x_{1}-a x_{4}, x_{2}, x_{3}\right)\right), x_{4}>0,\left(x_{1}, x_{2}, x_{3}\right) \in R^{3} .
\end{aligned}
$$

Thus, we have found an infinite set of solutions to the original problem, depending on the values of the parameters $p_{i}, q_{i}, i=1,2,3,4$ the components of which are expressed by formulas (16) to (19). To find the parameters $p_{i}, q_{i}, i=1,2,3,4$ compare conditions (12) to (15) with conditions (6). From this comparison for the parameters we obtain the system

$\frac{p_{1} q_{1}}{p_{2} q_{2}}=s_{12}, \frac{p_{1} q_{1}}{p_{3} q_{3}}=s_{13}, \frac{p_{1} q_{2}}{p_{2} q_{1}}=s_{21}, \frac{p_{1} q_{3}}{p_{3} q_{1}}=s_{31}, \frac{q_{1}}{q_{2}}=\frac{q_{3}}{q_{4}}$.

Solving the last system (20), we find

$$
\begin{aligned}
& \left(\frac{q_{1}}{q_{2}}\right)^{2}=\frac{s_{12}}{s_{21}},\left(\frac{q_{2}}{q_{3}}\right)^{2}=\frac{s_{21} s_{13}}{s_{12} s_{31}},\left(\frac{q_{1}}{q_{3}}\right)^{2}=\frac{s_{13}}{s_{31}},\left(\frac{q_{2}}{q_{4}}\right)^{2}=\frac{s_{13}}{s_{31}},\left(\frac{q_{1}}{q_{4}}\right)^{2}=\frac{s_{12} s_{13}}{s_{21} s_{31}},\left(\frac{q_{3}}{q_{4}}\right)^{2}=\frac{s_{12}}{s_{21}}, \\
& \left(\frac{p_{1}}{p_{2}}\right)^{2}=s_{12} s_{21},\left(\frac{p_{1}}{p_{3}}\right)^{2}=s_{13} s_{31},\left(\frac{p_{2}}{p_{3}}\right)^{2}=\frac{s_{13} s_{31}}{s_{12} s_{21}} .
\end{aligned}
$$

The ratio $\frac{p_{4}}{p_{1}}$ is determined from the condition (3):

$$
\frac{p_{4}}{p_{1}}=\mp \frac{s_{12} s_{13}+s_{12}+s_{13}}{\sqrt{s_{12} s_{21} s_{13} s_{31}}}=\mp \sqrt{\frac{s_{12} s_{13}}{s_{21} s_{31}}}\left(1+\frac{1}{s_{12}}+\frac{1}{s_{13}}\right) .
$$

Note that solutions (16) to (19) depend only on $\frac{p_{1}}{p_{4}}, \frac{q_{1}}{q_{4}}$ and $\frac{q_{2}}{q_{3}}$. Substituting the found ratios into formulas (16) to (19), we obtain two solutions to the original problem, but it is easy to see that these two solutions coincide, since $\operatorname{sign}\left(\frac{p_{1}}{p_{4}}\right) \operatorname{sign}\left(\frac{q_{1}}{q_{4}}\right)<0, \quad \operatorname{sign}\left(\frac{p_{1}}{p_{4}}\right) \operatorname{sign}\left(\frac{q_{2}}{q_{3}}\right)<0$, $\operatorname{sign}\left(\frac{q_{1}}{q_{4}}\right) \operatorname{sign}\left(\frac{q_{2}}{q_{3}}\right)>0$. The resulting solution is expressed by the formulas (7).

The found solution is the unique in the class $S_{5}^{1}$. Indeed, if there are two solutions, then their difference satisfies Eq. (1) with zero initial conditions. It is enough to prove that problem (1), (5) with $u_{10}=u_{20}=u_{30}=u_{40}=0$ has only the zero solution in the class $S_{5}^{1}$. If this problem has a nonzero solution, then its components must satisfy Eq. (9) with zero initial Cauchy condition. But such a problem cannot have a nonzero solution. Got a contradiction.

By direct verification, using conditions (3), (12) to (15) easy to make sure that the solution found satisfies Eq. (1) and the initial conditions (5).

The theorem is proved.

Comment: Conditions (6) are necessary and sufficient conditions for the existence and uniqueness of a solution to the Cauchy problem for Eq. (1).

\section{Results}

Let us move on to solving the filtration theory model. The continuity equation in filtration theory has the following form 
$m \frac{\partial \rho}{\partial t}+\operatorname{div}(\rho \vec{v})=0$,

Let us change the variables $(x, y, z, t)$ by $(x, y, z, \tau)$, where $\tau=c t, c$ is the characteristic velocity. Then the equation can be rewritten as follows

$c m \frac{\partial \rho}{\partial \tau}+\operatorname{div}(\rho \vec{v})=0$,

where $m=m(x, y, z)$ - known medium porosity, $\rho(x, y, z, t)$ - fluid density, $\vec{v}(x, \tau)=$ $\left(v_{1}(x, y, z, \tau), v_{2}(x, y, z, \tau), v_{3}(x, y, z, \tau)\right)$ - filtration velocity, $x=(x, y, z) \in R^{3}$ - spatial coordinates, $\tau \in R_{+}$- time.

Write the general nonlinear filtration law in the following form

$-\nabla p=\frac{v}{k} \vec{f}\left(\rho, v_{1}, v_{2}, v_{3}\right)$

where $v$ - kinematic fluid viscosity, $k=k(x, y, z)$ - permeability coefficient depending only on the properties of the porous medium, $p(x, y, z, \tau)-$ fluid pressure, $\vec{f}\left(\rho, v_{1}, v_{2}, v_{3}\right)-$ a given function of its arguments. Let us assume that the viscosity of the liquid and the coefficient of permeability are constant values.

For $\vec{f}\left(\rho, v_{1}, v_{2}, v_{3}\right)=\left(\rho v_{1}, \rho v_{2}, \rho v_{3}\right)$, Eq. (24) is called the linear Darcy law

$$
\left\{\begin{array}{l}
\frac{\partial p}{\partial x}=-\frac{v}{k} \rho v_{1} \\
\frac{\partial p}{\partial y}=-\frac{v}{k} \rho v_{2} \\
\frac{\partial p}{\partial z}=-\frac{v}{k} \rho v_{3} .
\end{array}\right.
$$

For $\quad \vec{f}\left(\rho, v_{1}, v_{2}, v_{3}\right)=\left(\rho^{2} v_{1}{ }^{2}-\rho^{2} v_{2}{ }^{2}-\rho^{2} v_{3}{ }^{2}+\rho^{2} c^{2} m^{2}, 2 \rho^{2} v_{3} c m-2 \rho^{2} v_{1} v_{2}, 2 \rho^{2} v_{2} c m-\right.$ $\left.2 \rho^{2} v_{1} v_{3}\right)$ Eq. (24) is called Forchheimer's nonlinear filtration law

$$
\left\{\begin{array}{l}
\frac{\partial p}{\partial x}=-\frac{v}{k}\left(\rho^{2} v_{1}^{2}-\rho^{2} v_{2}^{2}-\rho^{2} v_{3}^{2}+\rho^{2}(c m)^{2}\right), \\
\frac{\partial p}{\partial y}=-2 \frac{v}{k}\left(\rho^{2} v_{3} c m-\rho^{2} v_{1} v_{2}\right) \\
\frac{\partial p}{\partial z}=-2 \frac{v}{k}\left(\rho^{2} v_{2} c m-\rho^{2} v_{1} v_{3}\right)
\end{array}\right.
$$

Consider the Cauchy problem for system (23), (24)

$$
\begin{aligned}
& \rho(x, y, z, 0)=\rho_{0}(x, y, z), 0<\rho_{\text {min }} \leq \rho_{0}(x, y, z) \leq \rho_{\max }<\infty, \\
& v_{i}(x, y, z, 0)=\varphi_{i}(x, y, z),(x, y, z) \in R^{3}, i=1,2,3 .
\end{aligned}
$$

Suppose that the functions $\rho_{0}, \varphi_{1}, \varphi_{2}, \varphi_{3}$ tend to zero as the spatial coordinates tend to infinity. Let us introduce the following notation: $\rho(x, y, z, \tau) \cdot v_{1}(x, y, z, \tau)=u_{1}(x, y, z, \tau), \rho(x, y, z, t)$. $v_{2}(x, y, z, \tau)=u_{2}(x, y, z, \tau), \rho(x, y, z, \tau) \cdot v_{3}(x, y, z, \tau)=u_{3}(x, y, z, \tau), c m(x, y, z) \cdot \rho(x, y, z, \tau)=$ $u_{4}(x, y, z, \tau)$. Then Eq. (23) can be rewritten as follows 
$\operatorname{div} U=\frac{\partial u_{1}}{\partial x}+\frac{\partial u_{2}}{\partial y}+\frac{\partial u_{3}}{\partial z}+\frac{\partial u_{4}}{\partial \tau}=0$.

From (27), (28) for the functions $u_{1}, u_{2}, u_{3}, u_{4}$ we have the following initial conditions

$$
\begin{aligned}
& u_{1}(x, y, z, 0)=\rho_{0}(x, y, z) \varphi_{1}(x, y, z), \\
& u_{2}(x, y, z, 0)=\rho_{0}(x, y, z) \varphi_{2}(x, y, z), \\
& u_{3}(x, y, z, 0)=\rho_{0}(x, y, z) \varphi_{3}(x, y, z), \\
& u_{4}(x, y, z, 0)=c m(x, y, z) \rho_{0}(x, y, z) .
\end{aligned}
$$

According to Theorem 2, problem (29), (30) has a unique solution if the initial data satisfy the following conditions

$$
\left\{\begin{array}{c}
\frac{\partial\left(\rho_{0} \varphi_{1}\right)}{\partial x}=s_{12} \frac{\partial\left(\rho_{0} \varphi_{2}\right)}{\partial y}=s_{13} \frac{\partial\left(\rho_{0} \varphi_{3}\right)}{\partial z} \\
\frac{\partial\left(\rho_{0} \varphi_{2}\right)}{\partial x}=-s_{21} \frac{\partial\left(\rho_{0} \varphi_{1}\right)}{\partial y}=s_{13} \frac{\partial\left(c m \rho_{0}\right)}{\partial z} \\
\frac{\partial\left(\rho_{0} \varphi_{3}\right)}{\partial x}=s_{12} \frac{\partial\left(c m \rho_{0}\right)}{\partial y}=-s_{31} \frac{\partial\left(\rho_{0} \varphi_{1}\right)}{\partial z} \\
\frac{\partial\left(c m \rho_{0}\right)}{\partial x}=-s_{21} \frac{\partial\left(\rho_{0} \varphi_{3}\right)}{\partial y}=-s_{31} \frac{\partial\left(\rho_{0} \varphi_{2}\right)}{\partial z}
\end{array}\right.
$$

for $(x, y, z) \in R^{3}$, where $s_{12}, s_{13}, s_{24}, s_{21}, s_{31}$ given real constants. In this case, the solution of problem (29), (30) is

$$
\begin{aligned}
& u_{1}(x, y, z, \tau)=\frac{1}{2}\left[\rho_{0}\left(x+\frac{s_{12} s_{13}+s_{12}+s_{13}}{\sqrt{s_{12} s_{21} s_{13} s_{31}}} \tau, y, z\right) \varphi_{1}\left(x+\frac{s_{12} s_{13}+s_{12}+s_{13}}{\sqrt{s_{12} s_{21} s_{13} s_{31}}} \tau, y, z\right)+\rho_{0}(x-\right. \\
& \left.\frac{s_{12} s_{13}+s_{12}+s_{13}}{\sqrt{s_{12} s_{21} s_{13} s_{31}}} \tau, y, z\right) \varphi_{1}\left(x-\frac{s_{12} s_{13}+s_{12}+s_{13}}{\sqrt{s_{12} s_{21} s_{13} s_{31}}} \tau, y, z\right)+\sqrt{\frac{s_{12} s_{13}}{s_{21} s_{31}}}\left(c m \rho_{0}\left(x+\frac{s_{12} s_{13}+s_{12}+s_{13}}{\sqrt{s_{12} s_{21} s_{13} s_{31}}} \tau, y, z\right)-\right. \\
& \left.\left.c m \rho_{0}\left(x-\frac{s_{12} s_{13}+s_{12}+s_{13}}{\sqrt{s_{12} s_{21} s_{13} s_{31}}} \tau, y, z\right)\right)\right] \text {, } \\
& u_{2}(x, y, z, \tau)=\frac{1}{2}\left[\rho_{0}\left(x+\frac{s_{12} s_{13}+s_{12}+s_{13}}{\sqrt{s_{12} s_{21} s_{13} s_{31}}} \tau, y, z\right) \varphi_{2}\left(x+\frac{s_{12} s_{13}+s_{12}+s_{13}}{\sqrt{s_{12} s_{21} s_{13} s_{31}}} \tau, y, z\right)+\rho_{0}(x-\right. \\
& \left.\frac{s_{12} s_{13}+s_{12}+s_{13}}{\sqrt{s_{12} s_{21} s_{13} s_{31}}} \tau, y, z\right) \varphi_{2}\left(x-\frac{s_{12} s_{13}+s_{12}+s_{13}}{\sqrt{s_{12} s_{21} s_{13} s_{31}}} \tau, y, z\right)-\sqrt{\frac{s_{21} s_{13}}{s_{12} s_{31}}}\left(\rho_{0}\left(x+\frac{s_{12} s_{13}+s_{12}+s_{13}}{\sqrt{s_{12} s_{21} s_{13} s_{31}}} \tau, y, z\right) \varphi_{3}(x+\right. \\
& \left.\left.\left.\frac{s_{12} s_{13}+s_{12}+s_{13}}{\sqrt{s_{12} s_{21} s_{13} s_{31}}} \tau, y, z\right)-\rho_{0}\left(x-\frac{s_{12} s_{13}+s_{12}+s_{13}}{\sqrt{s_{12} s_{21} s_{13} s_{31}}} \tau, y, z\right) \varphi_{3}\left(x-\frac{s_{12} s_{13}+s_{12}+s_{13}}{\sqrt{s_{12} s_{21} s_{13} s_{31}}} \tau, y, z\right)\right)\right] \text {, } \\
& u_{3}(x, y, z, \tau)=\frac{1}{2}\left[\rho_{0}\left(x+\frac{s_{12} s_{13}+s_{12}+s_{13}}{\sqrt{s_{12} s_{21} s_{13} s_{31}}} \tau, y, z\right) \varphi_{3}\left(x+\frac{s_{12} s_{13}+s_{12}+s_{13}}{\sqrt{s_{12} s_{21} s_{13} s_{31}}} \tau, y, z\right)+\rho_{0}(x-\right. \\
& \left.\frac{s_{12} s_{13}+s_{12}+s_{13}}{\sqrt{s_{12} s_{21} s_{13} s_{31}}} \tau, y, z\right) \varphi_{3}\left(x-\frac{s_{12} s_{13}+s_{12}+s_{13}}{\sqrt{s_{12} s_{21} s_{13} s_{31}}} \tau, y, z\right)-\sqrt{\frac{s_{12} s_{31}}{s_{21} s_{13}}}\left(\rho_{0}\left(x+\frac{s_{12} s_{13}+s_{12}+s_{13}}{\sqrt{s_{12} s_{21} s_{13} s_{31}}} \tau, y, z\right) \varphi_{2}(x+\right. \\
& \left.\left.\left.\frac{s_{12} s_{13}+s_{12}+s_{13}}{\sqrt{s_{12} s_{21} s_{13} s_{31}}} \tau, y, z\right)-\rho_{0}\left(x-\frac{s_{12} s_{13}+s_{12}+s_{13}}{\sqrt{s_{12} s_{21} s_{13} s_{31}}} \tau, y, z\right) \varphi_{2}\left(x-\frac{s_{12} s_{13}+s_{12}+s_{13}}{\sqrt{s_{12} s_{21} s_{13} s_{31}}} \tau, y, z\right)\right)\right] \text {, }
\end{aligned}
$$




$$
\begin{aligned}
& u_{4}(x, y, z, \tau)=\frac{1}{2}\left[c m \rho_{0}\left(x+\frac{s_{12} s_{13}+s_{12}+s_{13}}{\sqrt{s_{12} s_{21} s_{13} s_{31}}} \tau, y, z\right)+m \rho_{0}\left(x-\frac{s_{12} s_{13}+s_{12}+s_{13}}{\sqrt{s_{12} s_{21} s_{13} s_{31}}} \tau, y, z\right)+\right. \\
& \sqrt{\frac{s_{21} s_{31}}{s_{12} s_{13}}}\left(\rho_{0}\left(x+\frac{s_{12} s_{13}+s_{12}+s_{13}}{\sqrt{s_{12} s_{21} s_{13} s_{31}}} \tau, y, z\right) \varphi_{1}\left(x+\frac{s_{12} s_{13}+s_{12}+s_{13}}{\sqrt{s_{12} s_{21} s_{13} s_{31}}} \tau, y, z\right)-\rho_{0}(x-\right. \\
& \left.\left.\left.\frac{s_{12} s_{13}+s_{12}+s_{13}}{\sqrt{s_{12} s_{21} s_{13} s_{31}}} \tau, y, z\right) \varphi_{1}\left(x-\frac{s_{12} s_{13}+s_{12}+s_{13}}{\sqrt{s_{12} s_{21} s_{13} s_{31}}} \tau, y, z\right)\right)\right] .
\end{aligned}
$$

From here we find the initial variables

$$
\begin{aligned}
& \rho(x, y, z, t)=\frac{1}{2 b c m(x, y, z)}\left(b\left(m \rho_{0}\right)^{+}+b\left(m \rho_{0}\right)^{-}+\left(\rho_{0} \varphi_{1}\right)^{+}-\left(\rho_{0} \varphi_{1}\right)^{-}\right), \\
& v_{1}(x, y, z, t)=b c m(x, y, z) \cdot \frac{\left(\rho_{0} \varphi_{1}\right)^{+}+\left(\rho_{0} \varphi_{1}\right)^{-}+b\left(m \rho_{0}\right)^{+}-b\left(m \rho_{0}\right)^{-}}{b\left(m \rho_{0}\right)^{+}+b\left(m \rho_{0}\right)^{-}+\left(\rho_{0} \varphi_{1}\right)^{+}-\left(\rho_{0} \varphi_{1}\right)^{-\prime}} \\
& v_{2}(x, y, z, t)=b c m(x, y, z) \cdot \frac{\left(\rho_{0} \varphi_{2}\right)^{+}+\left(\rho_{0} \varphi_{2}\right)^{-}-d\left(\rho_{0} \varphi_{3}\right)^{+}+d\left(\rho_{0} \varphi_{3}\right)^{-}}{b\left(m \rho_{0}\right)^{+}+b\left(m \rho_{0}\right)^{-}+\left(\rho_{0} \varphi_{1}\right)^{+}-\left(\rho_{0} \varphi_{1}\right)^{-}} \\
& v_{3}(x, y, z, t)=\frac{s_{12}}{s_{21}} \operatorname{cm}(x, y, z) \cdot \frac{d\left(\rho_{0} \varphi_{3}\right)^{+}+d\left(\rho_{0} \varphi_{3}\right)^{-}-\left(\rho_{0} \varphi_{2}\right)^{+}+\left(\rho_{0} \varphi_{2}\right)^{-}}{b\left(m \rho_{0}\right)^{+}+b\left(m \rho_{0}\right)^{-}+\left(\rho_{0} \varphi_{1}\right)^{+}-\left(\rho_{0} \varphi_{1}\right)^{-}}
\end{aligned}
$$

where $\left(\rho_{0} \varphi_{1}\right)^{+}=\rho_{0}(x+a \tau, y, z) \varphi_{1}(x+a \tau, y, z),\left(\rho_{0} \varphi_{1}\right)^{-}=\rho_{0}(x-a \tau, y, z) \varphi_{1}(x-a \tau, y, z)$, $\left(\rho_{0} \varphi_{2}\right)^{+}=\rho_{0}(x+a c t, y, z) \varphi_{2}(x+a c t, y, z), \quad\left(\rho_{0} \varphi_{2}\right)^{-}=\rho_{0}(x-a \tau, y, z) \varphi_{2}(x-a \tau, y, z)$, $\left(\rho_{0} \varphi_{3}\right)^{+}=\rho_{0}(x+a \tau, y, z) \varphi_{3}(x+a \tau, y, z), \quad\left(\rho_{0} \varphi_{3}\right)^{-}=\rho_{0}(x-a \tau, y, z) \varphi_{3}(x-a \tau, y, z)$, $\left(m \rho_{0}\right)^{+}=c m(x, y, z) \rho_{0}(x+a \tau, y, z), \quad\left(m \rho_{0}\right)^{-}=c m(x, y, z) \rho_{0}(x-a \tau, y, z)$, $a=\frac{s_{12} s_{13}+s_{12}+s_{13}}{\sqrt{s_{12} s_{21} s_{13} s_{31}}}, b=\sqrt{\frac{s_{12} s_{13}}{s_{21} s_{31}}}, d=\sqrt{\frac{s_{21} s_{13}}{s_{12} s_{31}}}$.

Thus, if conditions (31) are satisfied, then problem (23), (27), (28) has a unique solution (32) to (35). Substituting this solution into the equation of motion (24), we obtain the equation for the pressure. For the solvability of Eq. (24), it is necessary that

$\operatorname{rot}\left(\vec{f}\left(\rho, v_{1}, v_{2}, v_{3}\right)\right)=0$

This relation imposes a restriction on the form of the function $\vec{f}$. Define a function $\vec{f}$ by the following theorem.

Let $h(x, y, z, \tau)$ be an arbitrary four-dimensional regular function and denote by $\vec{g}(x, y, z, \tau)$ a four-dimensional function with components

$$
\left\{\begin{array}{l}
g_{1}(x, y, z, \tau)=r_{1} h_{1}\left(s_{1} x, s_{2} y, s_{3} z, s_{4} \tau\right) \\
g_{2}(x, y, z, \tau)=r_{2} h_{2}\left(s_{1} x, s_{2} y, s_{3} z, s_{4} \tau\right) \\
g_{3}(x, y, z, \tau)=r_{3} h_{3}\left(s_{1} x, s_{2} y, s_{3} z, s_{4} \tau\right)^{\prime} \\
g_{4}(x, y, z, \tau)=r_{4} h_{4}\left(s_{1} x, s_{2} y, s_{3} z, s_{4} \tau\right)
\end{array}\right.
$$

where $r_{i}, s_{i}, i=1,2,3,4$, - definable constants. Then for $\vec{f}$ we take the following function with components 


$$
\left\{\begin{array}{c}
f_{1}\left(\rho, v_{1}, v_{2}, v_{3}\right)=g_{1}\left(\rho v_{1}, \rho v_{2}, \rho v_{3}, m \rho\right)-g_{1}(0,0,0,0)= \\
=r_{1} h_{1}\left(s_{1} \rho v_{1}, s_{2} \rho v_{2}, s_{3} \rho v_{3}, s_{4} m \rho\right)-r_{1} h_{1}(0,0,0,0) \\
f_{2}\left(\rho, v_{1}, v_{2}, v_{3}\right)=g_{2}\left(\rho v_{1}, \rho v_{2}, \rho v_{3}, m \rho\right)-g_{2}(0,0,0,0)= \\
=r_{2} h_{2}\left(s_{1} \rho v_{1}, s_{2} \rho v_{2}, s_{3} \rho v_{3}, s_{4} m \rho\right)-r_{2} h_{2}(0,0,0,0) \\
f_{3}\left(\rho, v_{1}, v_{2}, v_{3}\right)=g_{3}\left(\rho v_{1}, \rho v_{2}, \rho v_{3}, m \rho\right)= \\
=r_{3} h_{3}\left(s_{1} \rho v_{1}, s_{2} \rho v_{2}, s_{3} \rho v_{3}, s_{4} m \rho\right)
\end{array}\right.
$$

The function $\vec{f}$ from (37) must satisfy the following condition

$\operatorname{rot} \vec{f}(x, y, z, \tau)=\left(\begin{array}{l}\frac{\partial f_{3}}{\partial y}-\frac{\partial f_{2}}{\partial z} \\ \frac{\partial f_{1}}{\partial z}-\frac{\partial f_{3}}{\partial x} \\ \frac{\partial f_{2}}{\partial x}-\frac{\partial f_{1}}{\partial y}\end{array}\right)=0$

for all $\tau \in R_{+},(x, y, z) \in R^{3}$.

Theorem 3: (Darcy's Law) The Cauchy problem for the mathematical model of the linear filtration theory (23), (25), (27), (28) satisfying conditions (29), with $s_{12}=s_{21}=s_{13}=s_{31}=1$, has a unique solution

$$
\begin{aligned}
& \rho(x, y, z, \tau)=\frac{1}{2 c m(x, y, z)}\left(\left(m \rho_{0}\right)^{+}+\left(m \rho_{0}\right)^{-}+\left(\rho_{0} \varphi_{1}\right)^{+}-\left(\rho_{0} \varphi_{1}\right)^{-}\right) \\
& v_{1}(x, y, z, \tau)=c m(x, y, z) \cdot \frac{\left(\rho_{0} \varphi_{1}\right)^{+}+\left(\rho_{0} \varphi_{1}\right)^{-}+\left(m \rho_{0}\right)^{+}-\left(m \rho_{0}\right)^{-}}{\left(m \rho_{0}\right)^{+}+\left(m \rho_{0}\right)^{-}+\left(\rho_{0} \varphi_{1}\right)^{+}-\left(\rho_{0} \varphi_{1}\right)^{-}} \\
& v_{2}(x, y, z, \tau)=c m(x, y, z) \cdot \frac{\left(\rho_{0} \varphi_{2}\right)^{+}+\left(\rho_{0} \varphi_{2}\right)^{-}-\left(\rho_{0} \varphi_{3}\right)^{+}+\left(\rho_{0} \varphi_{3}\right)^{-}}{\left(m \rho_{0}\right)^{+}+\left(m \rho_{0}\right)^{-}+\left(\rho_{0} \varphi_{1}\right)^{+}-\left(\rho_{0} \varphi_{1}\right)^{-}} \\
& v_{3}(x, y, z, \tau)=c m(x, y, z) \cdot \frac{\left(\rho_{0} \varphi_{3}\right)^{+}+\left(\rho_{0} \varphi_{3}\right)^{-}-\left(\rho_{0} \varphi_{2}\right)^{+}+\left(\rho_{0} \varphi_{2}\right)^{-}}{\left(m \rho_{0}\right)^{+}+\left(m \rho_{0}\right)^{-}+\left(\rho_{0} \varphi_{1}\right)^{+}-\left(\rho_{0} \varphi_{1}\right)^{-}} \\
& p=-\frac{\mu}{k} \int_{0}^{x} v_{1}(\xi, y, z, \tau) d \xi-\frac{\mu}{k} \int_{0}^{y} v_{2}(0, \eta, z, \tau) d \eta-\frac{\mu}{k} \int_{0}^{z} v_{3}(0,0, \zeta, \tau) d \zeta+C(\tau)
\end{aligned}
$$

where

$$
\begin{array}{ll}
\left(\rho_{0} \varphi_{1}\right)^{+}=\rho_{0}(x+3 \tau, y, z) \varphi_{1}((x+3 \tau, y, z), & \left(\rho_{0} \varphi_{1}\right)^{-}=\rho_{0}(x-3 \tau, y, z) \varphi_{1}(x-3 \tau, y, z), \\
\left(\rho_{0} \varphi_{2}\right)^{+}=\rho_{0}\left((x+3 \tau, y, z) \varphi_{2}((x+3 \tau, y, z),\right. & \left(\rho_{0} \varphi_{2}\right)^{-}=\rho_{0}(x-3 \tau, y, z) \varphi_{2}(x-3 \tau, y, z), \\
\left(\rho_{0} \varphi_{3}\right)^{+}=\rho_{0}\left((x+3 \tau, y, z) \varphi_{3}((x+3 \tau, y, z),\right. & \left(\rho_{0} \varphi_{3}\right)^{-}=\rho_{0}(x-3 \tau, y, z) \varphi_{3}(x-3 \tau, y, z), \\
\left(m \rho_{0}\right)^{+}=c m(x, y, z) \rho_{0}((x+3 \tau, y, z), & \left(m \rho_{0}\right)^{-}=c m(x, y, z) \rho_{0}(x-3 \tau, y, z) .
\end{array}
$$

Proof: Substituting the given coefficients $s_{12}=s_{21}=s_{13}=s_{31}=1$ into (32) to (35), we find

$$
\begin{aligned}
& \rho(x, y, z, \tau)=\frac{1}{2 c m(x, y, z)}\left(\left(m \rho_{0}\right)^{+}+\left(m \rho_{0}\right)^{-}+\left(\rho_{0} \varphi_{1}\right)^{+}-\left(\rho_{0} \varphi_{1}\right)^{-}\right) \\
& v_{1}(x, y, z, \tau)=c m(x, y, z) \cdot \frac{\left(\rho_{0} \varphi_{1}\right)^{+}+\left(\rho_{0} \varphi_{1}\right)^{-}+\left(m \rho_{0}\right)^{+}-\left(m \rho_{0}\right)^{-}}{\left(m \rho_{0}\right)^{+}+\left(m \rho_{0}\right)^{-}+\left(\rho_{0} \varphi_{1}\right)^{+}-\left(\rho_{0} \varphi_{1}\right)^{-}} \\
& v_{2}(x, y, z, \tau)=c m(x, y, z) \cdot \frac{\left(\rho_{0} \varphi_{2}\right)^{+}+\left(\rho_{0} \varphi_{2}\right)^{-}-\left(\rho_{0} \varphi_{3}\right)^{+}+\left(\rho_{0} \varphi_{3}\right)^{-}}{\left(m \rho_{0}\right)^{+}+\left(m \rho_{0}\right)^{-}+\left(\rho_{0} \varphi_{1}\right)^{+}-\left(\rho_{0} \varphi_{1}\right)^{-}}
\end{aligned}
$$


$v_{3}(x, y, z, \tau)=c m(x, y, z) \cdot \frac{\left(\rho_{0} \varphi_{3}\right)^{+}+\left(\rho_{0} \varphi_{3}\right)^{-}-\left(\rho_{0} \varphi_{2}\right)^{+}+\left(\rho_{0} \varphi_{2}\right)^{-}}{\left(m \rho_{0}\right)^{+}+\left(m \rho_{0}\right)^{-}+\left(\rho_{0} \varphi_{1}\right)^{+}-\left(\rho_{0} \varphi_{1}\right)^{-}}$

where $\left(\rho_{0} \varphi_{1}\right)^{+}=\rho_{0}(x+3 \tau, y, z) \varphi_{1}\left((x+3 \tau, y, z), \quad\left(\rho_{0} \varphi_{1}\right)^{-}=\rho_{0}(x-3 \tau, y, z) \varphi_{1}(x-\right.$ $3 \tau, y, z)$,

$\left(\rho_{0} \varphi_{2}\right)^{+}=\rho_{0}\left((x+3 \tau, y, z) \varphi_{2}\left((x+3 \tau, y, z), \quad\left(\rho_{0} \varphi_{2}\right)^{-}=\rho_{0}(x-3 \tau, y, z) \varphi_{2}(x-3 \tau, y, z)\right.\right.$, $\left(\rho_{0} \varphi_{3}\right)^{+}=\rho_{0}\left((x+3 \tau, y, z) \varphi_{3}\left((x+3 \tau, y, z), \quad\left(\rho_{0} \varphi_{3}\right)^{-}=\rho_{0}(x-3 \tau, y, z) \varphi_{3}(x-3 \tau, y, z)\right.\right.$, $\left(m \rho_{0}\right)^{+}=c m(x, y, z) \rho_{0}\left((x+3 \tau, y, z), \quad\left(m \rho_{0}\right)^{-}=c m(x, y, z) \rho_{0}(x-3 \tau, y, z)\right.$.

Let us choose a linear function $h(x, y, z, \tau)=X$, then for $s_{12}=s_{21}=s_{13}=s_{31}=1$ we obtain $f_{1}\left(\rho, v_{1}, v_{2}, v_{3}\right)=\rho v_{1}, f_{2}\left(\rho, v_{1}, v_{2}, v_{3}\right)=\rho v_{2}, f_{3}\left(\rho, v_{1}, v_{2}, v_{3}\right)=\rho v_{3}$

Check the condition (38)

$$
\begin{aligned}
& \frac{\partial f_{3}}{\partial y}-\frac{\partial f_{2}}{\partial z}=\frac{\partial\left(\rho v_{3}\right)}{\partial y}-\frac{\partial\left(\rho v_{2}\right)}{\partial z}=\frac{\partial\left(\rho v_{3}\right)}{\partial y}-\frac{s_{21}}{s_{31}} \frac{\partial\left(\rho v_{3}\right)}{\partial y}=0 \\
& \frac{\partial f_{1}}{\partial z}-\frac{\partial f_{3}}{\partial x}=-\frac{\partial\left(\rho v_{1}\right)}{\partial z}-\frac{\partial\left(\rho v_{3}\right)}{\partial x}=-\frac{\partial\left(\rho v_{1}\right)}{\partial z}+s_{31} \frac{\partial\left(\rho v_{1}\right)}{\partial z}=0 \\
& \frac{\partial f_{2}}{\partial x}-\frac{\partial f_{1}}{\partial y}=\frac{\partial\left(\rho v_{2}\right)}{\partial x}+\frac{\partial\left(\rho v_{1}\right)}{\partial y}=\frac{\partial\left(\rho v_{2}\right)}{\partial x}-\frac{1}{s_{21}} \frac{\partial\left(\rho v_{2}\right)}{\partial x}=0 .
\end{aligned}
$$

Thus, Eq. (37) is satisfied. Therefore, there exists a scalar function $\psi(x, y, z, \tau)$ such that

$\vec{f}(x, y, z, \tau)=\nabla \psi(x, y, z, \tau)$

Such a function $\psi(x, y, z, \tau)$ has the form

$\psi(x, y, z, \tau)=\int_{0}^{x}\left(f_{1}\left(\rho v_{1}, \rho v_{2}, \rho v_{3}, m \rho\right)\right)(\xi, y, z, \tau) d \xi+\int_{0}^{y}\left(f_{2}\left(\rho v_{1}, \rho v_{2}, \rho v_{3}, m \rho\right)\right)(0, \eta, z, \tau) d \eta+$ $\int_{0}^{z}\left(f_{3}\left(\rho v_{1}, \rho v_{2}, \rho v_{3}, m \rho\right)\right)(0,0, \zeta, \tau) d \zeta+C(v)$.

Then, Eq. (25) can be written as

$-\nabla p=\nabla\left(\frac{v}{k} \psi\right)$

which has a solution

$$
\left\{\begin{array}{l}
\frac{\partial p}{\partial x}=-\frac{v}{k} \rho f_{1}\left(v_{1}, v_{2}, v_{3}\right)=-\frac{v}{k} f_{1}\left(\rho, v_{1}, v_{2}, v_{3}\right)=-\frac{v}{k} \rho v_{1}=-\frac{\mu}{k} v_{1} \\
\frac{\partial p}{\partial y}=-\frac{v}{k} \rho f_{2}\left(v_{1}, v_{2}, v_{3}\right)=-\frac{v}{k} f_{2}\left(\rho, v_{1}, v_{2}, v_{3}\right)=-\frac{v}{k} \rho v_{2}=-\frac{\mu}{k} v_{2} \\
\frac{\partial p}{\partial z}=-\frac{v}{k} \rho f_{3}\left(v_{1}, v_{2}, v_{3}\right)=-\frac{v}{k} f_{3}\left(\rho, v_{1}, v_{2}, v_{3}\right)=-\frac{v}{k} \rho v_{3}=-\frac{\mu}{k} v_{3}
\end{array}\right.
$$

Calculating we get a solution for the pressure 
$p=-\frac{\mu}{k} \int_{0}^{x} v_{1}(\xi, y, z, \tau) d \xi-\frac{\mu}{k} \int_{0}^{y} v_{2}(0, \eta, z, \tau) d \eta-\frac{\mu}{k} \int_{0}^{z} v_{3}(0,0, \zeta, \tau) d \zeta+C(\tau)$

To find $C(\tau)$, we use the fact that as the spatial variables tend to infinity, the filtration rate and density tend to zero. Then

$p_{\infty}=-\frac{\mu}{k} \int_{0}^{\infty} v_{3}(0,0, \zeta, \tau) d \zeta+C(\tau)$

whence

$C(\tau)=p_{\infty}+\frac{\mu}{k} \int_{0}^{\infty} v_{3}(0,0, \zeta, \tau) d \zeta$

The theorem is proved.

Thus, we obtained an explicit analytical solution to the three-dimensional mathematical model of linear filtration (39) to (43).

Theorem 4: (Second order nonlinear filtration law) The Cauchy problem for a nonlinear mathematical model of filtration theory (23), (26), (27), (28) satisfying conditions (29), for $s_{12}=$ $s_{21}=s_{13}=s_{31}=1$, has a unique solution

$\rho(x, y, z, \tau)=\frac{1}{2 c m(x, y, z)}\left(\left(m \rho_{0}\right)^{+}+\left(m \rho_{0}\right)^{-}+\left(\rho_{0} \varphi_{1}\right)^{+}-\left(\rho_{0} \varphi_{1}\right)^{-}\right)$

$v_{1}(x, y, z, \tau)=\operatorname{cm}(x, y, z) \cdot \frac{\left(\rho_{0} \varphi_{1}\right)^{+}+\left(\rho_{0} \varphi_{1}\right)^{-}+\left(m \rho_{0}\right)^{+}-\left(m \rho_{0}\right)^{-}}{\left(m \rho_{0}\right)^{+}+\left(m \rho_{0}\right)^{-}+\left(\rho_{0} \varphi_{1}\right)^{+}-\left(\rho_{0} \varphi_{1}\right)^{-}}$

$v_{2}(x, y, z, \tau)=\operatorname{cm}(x, y, z) \cdot \frac{\left(\rho_{0} \varphi_{2}\right)^{+}+\left(\rho_{0} \varphi_{2}\right)^{-}-\left(\rho_{0} \varphi_{3}\right)^{+}+\left(\rho_{0} \varphi_{3}\right)^{-}}{\left(m \rho_{0}\right)^{+}+\left(m \rho_{0}\right)^{-}+\left(\rho_{0} \varphi_{1}\right)^{+}-\left(\rho_{0} \varphi_{1}\right)^{-}}$

$v_{3}(x, y, z, \tau)=\operatorname{cm}(x, y, z) \cdot \frac{\left(\rho_{0} \varphi_{3}\right)^{+}+\left(\rho_{0} \varphi_{3}\right)^{-}-\left(\rho_{0} \varphi_{2}\right)^{+}+\left(\rho_{0} \varphi_{2}\right)^{-}}{\left(m \rho_{0}\right)^{+}+\left(m \rho_{0}\right)^{-}+\left(\rho_{0} \varphi_{1}\right)^{+}-\left(\rho_{0} \varphi_{1}\right)^{-}}$

$p=-\frac{\mu}{k} \int_{0}^{x} \rho\left(v_{1}^{2}-v_{2}^{2}-v_{3}^{2}+(\mathrm{cm})^{2}\right) d \xi-2 \frac{\mu}{k} \int_{0}^{y} \rho\left(v_{3} c m-v_{1} v_{2}\right) d \eta-2 \frac{\mu}{k} \int_{0}^{z} \rho\left(v_{2} c m-\right.$ $\left.v_{1} v_{3}\right) d \zeta+C(t)$

where $\left(\rho_{0} \varphi_{1}\right)^{+}=\rho_{0}(x+3 \tau, y, z) \varphi_{1}\left((x+3 \tau, y, z), \quad\left(\rho_{0} \varphi_{1}\right)^{-}=\rho_{0}(x-3 \tau, y, z) \varphi_{1}(x-3 \tau, y, z)\right.$, $\left(\rho_{0} \varphi_{2}\right)^{+}=\rho_{0}\left((x+3 \tau, y, z) \varphi_{2}\left((x+3 \tau, y, z), \quad\left(\rho_{0} \varphi_{2}\right)^{-}=\rho_{0}(x-3 \tau, y, z) \varphi_{2}(x-3 \tau, y, z)\right.\right.$, $\left(\rho_{0} \varphi_{3}\right)^{+}=\rho_{0}\left((x+3 \tau, y, z) \varphi_{3}\left((x+3 \tau, y, z), \quad\left(\rho_{0} \varphi_{3}\right)^{-}=\rho_{0}(x-3 \tau, y, z) \varphi_{3}(x-3 \tau, y, z)\right.\right.$, $\left(m \rho_{0}\right)^{+}=c m(x, y, z) \rho_{0}((x+3 \tau, y, z)$, $\left(m \rho_{0}\right)^{-}=c m(x, y, z) \rho_{0}(x-3 \tau, y, z)$.

Proof: Substituting the given coefficients $s_{12}=s_{21}=s_{13}=s_{31}=1$ into (32) to (35), we find

$$
\begin{aligned}
& \rho(x, y, z, \tau)=\frac{1}{2 c m(x, y, z)}\left(\left(m \rho_{0}\right)^{+}+\left(m \rho_{0}\right)^{-}+\left(\rho_{0} \varphi_{1}\right)^{+}-\left(\rho_{0} \varphi_{1}\right)^{-}\right) \\
& v_{1}(x, y, z, \tau)=c m(x, y, z) \cdot \frac{\left(\rho_{0} \varphi_{1}\right)^{+}+\left(\rho_{0} \varphi_{1}\right)^{-}+\left(m \rho_{0}\right)^{+}-\left(m \rho_{0}\right)^{-}}{\left(m \rho_{0}\right)^{+}+\left(m \rho_{0}\right)^{-}+\left(\rho_{0} \varphi_{1}\right)^{+}-\left(\rho_{0} \varphi_{1}\right)^{-}}
\end{aligned}
$$


$v_{2}(x, y, z, \tau)=c m(x, y, z) \cdot \frac{\left(\rho_{0} \varphi_{2}\right)^{+}+\left(\rho_{0} \varphi_{2}\right)^{-}-\left(\rho_{0} \varphi_{3}\right)^{+}+\left(\rho_{0} \varphi_{3}\right)^{-}}{\left(m \rho_{0}\right)^{+}+\left(m \rho_{0}\right)^{-}+\left(\rho_{0} \varphi_{1}\right)^{+}-\left(\rho_{0} \varphi_{1}\right)^{-}}$,
$v_{3}(x, y, z, \tau)=c m(x, y, z) \cdot \frac{\left(\rho_{0} \varphi_{3}\right)^{+}+\left(\rho_{0} \varphi_{3}\right)^{-}-\left(\rho_{0} \varphi_{2}\right)^{+}+\left(\rho_{0} \varphi_{2}\right)^{-}}{\left(m \rho_{0}\right)^{+}+\left(m \rho_{0}\right)^{-}+\left(\rho_{0} \varphi_{1}\right)^{+}-\left(\rho_{0} \varphi_{1}\right)^{-}}$

where $\left(\rho_{0} \varphi_{1}\right)^{+}=\rho_{0}(x+3 \tau, y, z) \varphi_{1}\left((x+3 \tau, y, z), \quad\left(\rho_{0} \varphi_{1}\right)^{-}=\rho_{0}(x-3 \tau, y, z) \varphi_{1}(x-\right.$ $3 \tau, y, z)$,

$\left(\rho_{0} \varphi_{2}\right)^{+}=\rho_{0}\left((x+3 \tau, y, z) \varphi_{2}\left((x+3 \tau, y, z), \quad\left(\rho_{0} \varphi_{2}\right)^{-}=\rho_{0}(x-3 \tau, y, z) \varphi_{2}(x-3 \tau, y, z)\right.\right.$,

$\left(\rho_{0} \varphi_{3}\right)^{+}=\rho_{0}\left((x+3 \tau, y, z) \varphi_{3}\left((x+3 \tau, y, z), \quad\left(\rho_{0} \varphi_{3}\right)^{-}=\rho_{0}(x-3 \tau, y, z) \varphi_{3}(x-3 \tau, y, z)\right.\right.$,

$\left(m \rho_{0}\right)^{+}=c m(x, y, z) \rho_{0}\left((x+3 \tau, y, z), \quad\left(m \rho_{0}\right)^{-}=c m(x, y, z) \rho_{0}(x-3 \tau, y, z)\right.$.

Let us choose a nonlinear function $h(x, y, z, \tau)=X^{2}$, then for $s_{12}=s_{21}=s_{13}=s_{31}=1$ we obtain $f_{1}\left(\rho, v_{1}, v_{2}, v_{3}\right)=\rho^{2} v_{1}{ }^{2}-\rho^{2} v_{2}{ }^{2}-\rho^{2} v_{3}{ }^{2}+\rho^{2} c^{2} m^{2}, \quad f_{2}\left(\rho, v_{1}, v_{2}, v_{3}\right)=2 \rho^{2} v_{3} c m-$ $2 \rho^{2} v_{1} v_{2}, f_{3}\left(\rho, v_{1}, v_{2}, v_{3}\right)=2 \rho^{2} v_{2} c m-2 \rho^{2} v_{1} v_{3}$

Check the condition (38)

$$
\begin{aligned}
& \frac{\partial f_{3}}{\partial y}-\frac{\partial f_{2}}{\partial z}=\frac{\partial\left(-2 \rho^{2} v_{1} v_{3}+2 \rho^{2} v_{2} c m\right)}{\partial y}-\frac{\partial\left(2 \rho^{2} v_{3} c m-2 \rho^{2} v_{1} v_{2}\right)}{\partial z} \\
& =-2 \rho v_{1} \frac{\partial\left(\rho v_{3}\right)}{\partial y}-2 \rho v_{1} \frac{\partial\left(\rho v_{1}\right)}{\partial y}+2 \rho v_{2} \frac{\partial(c m \rho)}{\partial y}+2 c m \rho \frac{\partial\left(\rho v_{2}\right)}{\partial y}-2 \rho v_{3} \frac{\partial(\mathrm{cm} \rho)}{\partial z} \\
& -2 c m \rho \frac{\partial\left(\rho v_{3}\right)}{\partial z}+2 \rho v_{1} \frac{\partial\left(\rho v_{2}\right)}{\partial z}+2 \rho v_{2} \frac{\partial\left(\rho v_{1}\right)}{\partial z} \\
& =-2 \rho v_{1} \frac{\partial\left(\rho v_{3}\right)}{\partial y}-2 \rho v_{1} \frac{\partial\left(\rho v_{1}\right)}{\partial y}+2 \rho v_{2} \frac{\partial(\mathrm{cm} \rho)}{\partial y}+2 c m \rho \frac{\partial\left(\rho v_{2}\right)}{\partial y} \\
& +2 \frac{s_{21}}{s_{13}} \rho v_{3} \frac{\partial\left(\rho v_{1}\right)}{\partial y}-2 \frac{s_{12}}{s_{13}} c m \rho \frac{\partial\left(\rho v_{2}\right)}{\partial y}+2 \frac{s_{21}}{s_{31}} \rho v_{1} \frac{\partial\left(\rho v_{3}\right)}{\partial y}-2 \frac{s_{12}}{s_{31}} \rho v_{2} \frac{\partial(c m \rho)}{\partial y} \\
& =0 \text {, } \\
& \frac{\partial f_{1}}{\partial z}-\frac{\partial f_{3}}{\partial x}=\frac{\partial\left(\rho^{2} v_{1}{ }^{2}-\rho^{2} v_{2}{ }^{2}-\rho^{2} v_{3}{ }^{2}-\rho^{2} c^{2} m^{2}\right)}{\partial z}-\frac{\partial\left(2 \rho^{2} v_{2} c m-2 \rho^{2} v_{1} v_{3}\right)}{\partial x} \\
& =2 \rho v_{1} \frac{\partial\left(\rho v_{1}\right)}{\partial z}-2 \rho v_{2} \frac{\partial\left(\rho v_{2}\right)}{\partial z}-2 \rho v_{3} \frac{\partial\left(\rho v_{3}\right)}{\partial z}+2 c m \rho \frac{\partial(\mathrm{cm} \rho)}{\partial z}-2 \rho v_{2} \frac{\partial(\mathrm{cm} \rho)}{\partial x} \\
& -2 c m \rho \frac{\partial\left(\rho v_{2}\right)}{\partial x}+2 \rho v_{1} \frac{\partial\left(\rho v_{3}\right)}{\partial x}+2 \rho v_{3} \frac{\partial\left(\rho v_{1}\right)}{\partial x} \\
& =2 \rho v_{1} \frac{\partial\left(\rho v_{1}\right)}{\partial z}-2 \rho v_{2} \frac{\partial\left(\rho v_{2}\right)}{\partial z}-2 \rho v_{3} \frac{\partial\left(\rho v_{3}\right)}{\partial z}+2 c m \rho \frac{\partial(c m \rho)}{\partial z}+2 \rho v_{2} \frac{\partial\left(\rho v_{2}\right)}{\partial z} \\
& -2 s_{13} c m \rho \frac{\partial(\mathrm{cm} \rho)}{\partial z}-2 s_{31} \rho v_{1} \frac{\partial\left(\rho v_{1}\right)}{\partial z}+2 s_{13} \rho v_{3} \frac{\partial\left(\rho v_{3}\right)}{\partial z}=0 \text {, } \\
& \frac{\partial f_{2}}{\partial x}-\frac{\partial f_{1}}{\partial y}=\frac{\partial\left(2 \rho^{2} v_{3} c m-2 \rho^{2} v_{1} v_{2}\right)}{\partial x}-\frac{\partial\left(\rho^{2} v_{1}^{2}-\rho^{2} v_{2}{ }^{2}-\rho^{2} v_{3}{ }^{2}+\rho^{2} c^{2} m^{2}\right)}{\partial y} \\
& =2 \rho v_{3} \frac{\partial(c m \rho)}{\partial x}+2 c m \rho \frac{\partial\left(\rho v_{3}\right)}{\partial x}-2 \rho v_{1} \frac{\partial\left(\rho v_{2}\right)}{\partial x}-2 \rho v_{2} \frac{\partial\left(\rho v_{1}\right)}{\partial x}-2 \rho v_{1} \frac{\partial\left(\rho v_{1}\right)}{\partial y} \\
& +2 \rho v_{2} \frac{\partial\left(\rho v_{2}\right)}{\partial y}+2 \rho v_{3} \frac{\partial\left(\rho v_{3}\right)}{\partial y}-2 c m \rho \frac{\partial(\mathrm{cm} \rho)}{\partial y} \\
& =2 \rho v_{3} \frac{\partial(\mathrm{cm} \rho)}{\partial x}+2 c m \rho \frac{\partial\left(\rho v_{3}\right)}{\partial x}-2 \rho v_{1} \frac{\partial\left(\rho v_{2}\right)}{\partial x}-2 \rho v_{2} \frac{\partial\left(\rho v_{1}\right)}{\partial x}+2 \frac{1}{s_{21}} \rho v_{1} \frac{\partial\left(\rho v_{2}\right)}{\partial x} \\
& +2 \frac{1}{s_{12}} \rho v_{2} \frac{\partial\left(\rho v_{1}\right)}{\partial x}-2 \frac{1}{s_{21}} \rho v_{3} \frac{\partial(c m \rho)}{\partial x}-2 \frac{1}{s_{12}} c m \rho \frac{\partial\left(\rho v_{3}\right)}{\partial x}=0
\end{aligned}
$$


Thus, Eq. (38) is satisfied. Therefore, there exists a scalar function $\psi(x, y, z, \tau)$ such (44). Then Eq. (26) has a solution of

$$
\left\{\begin{array}{l}
\frac{\partial p}{\partial x}=-\frac{v}{k} \rho^{2}\left(v_{1}^{2}-v_{2}^{2}-v_{3}^{2}+(\mathrm{cm})^{2}\right), \\
\frac{\partial p}{\partial y}=-2 \frac{v}{k} \rho^{2}\left(v_{3} c m-v_{1} v_{2}\right), \\
\frac{\partial p}{\partial z}=-2 \frac{v}{k} \rho^{2}\left(v_{2} c m-v_{1} v_{3}\right) .
\end{array}\right.
$$

Calculating we get a solution for the pressure

$$
\begin{gathered}
p=-\frac{\mu}{k} \int_{0}^{x} \rho\left(v_{1}{ }^{2}-v_{2}{ }^{2}-v_{3}{ }^{2}+(c m)^{2}\right)(\xi, y, z, \tau) d \xi-2 \frac{\mu}{k} \int_{0}^{y} \rho\left(v_{3} c m-v_{1} v_{2}\right)(0, \eta, z, \tau) d \eta \\
-2 \frac{\mu}{k} \int_{0}^{z} \rho\left(v_{2} c m-v_{1} v_{3}\right)(0,0, \zeta, \tau) d \zeta+C(\tau)
\end{gathered}
$$

Where

$C(t)=p_{\infty}+\frac{\mu}{k} \int_{0}^{\infty} \rho\left(v_{2} c m-v_{1} v_{3}\right)(0,0, \zeta, \tau) d \zeta$

The theorem is proved.

Thus, an explicit analytical solution (45) to (49) of the three-dimensional mathematical nonlinear filtration model was obtained.

Theorem 5: The function $\rho(x, y, z, t)$ defined by equality (32) is a strictly positive function.

Proof: Rewrite an Eq. (23) as

$\frac{\partial(m \rho)}{\partial t}+\nabla(m \rho) \cdot \frac{\vec{v}}{m}+m \rho \cdot \operatorname{div}\left(\frac{\vec{v}}{m}\right)=0$,

which functions (32) to (35) satisfy. Divide both sides of this equation by $m \rho$ :

$\frac{\partial \ln (m \rho)}{\partial t}+\nabla \ln (m \rho) \cdot \vec{u}=-\operatorname{div} \vec{u}$

where $\vec{u}=\left(u_{1}, u_{2}, u_{3}\right)=\frac{\vec{v}}{m}$. Eq. (36) for the function $\ln (m \rho)$ with the initial conditions $\ln (m \rho)(x, y, z, 0)=\left(m \rho_{0}\right)(x, y, z)$ has a unique solution $\sigma(x, y, z, t)$. Then $\rho(x, y, z, t)=$ $\frac{\exp (\sigma(x, y, z, t))}{m(x, y, z)}>0$ and, since the solution is unique, it coincides with the function (29).

The theorem is proved.

\section{Conclusion}

In this paper, a mathematical model of filtration theory was investigated and the existence and uniqueness of a solution to the Cauchy problem for this model were proved. An analytical solution 
of a three-dimensional problem is obtained by the method of four-dimensional mathematics in the Abenov space M5 for the linear Darcy law and the second order nonlinear filtration law.

\section{References}

[1] Nield, Donald A., and Adrian Bejan. Convection in porous media. Vol. 3. New York: springer, 2006.

[2] Coutelieris, Frank A., and J. M. P. Q. Delgado. "Modeling of Transport Processes in Porous Materials." In Transport Processes in Porous Media, pp. 87-121. Springer, Berlin, Heidelberg, 2012. https://doi.org/10.1007/978-3-642$\underline{27910-2 \quad 5}$

[3] Coussy, Olivier. Mechanics and physics of porous solids. John Wiley \& Sons, 2011. https://doi.org/10.1002/9780470710388

[4] Bear, Jacob, and M. Yavuz Corapcioglu, eds. Fundamentals of transport phenomena in porous media. Springer Science \& Business Media, 1972.

[5] Vafai, Kambiz, ed. Handbook of Porous Media. CRC Press, 2005. https://doi.org/10.1201/9780415876384

[6] Entov, V. M. "Filtration theory." Sorosov. Obrazovat. Zh 2 (1998): 121-128.

[7] Polubarinova-Koch, Pelageia lakovlevna. Theory of groundwater movement. Princeton University Press, 1977.

[8] Dullien, Francis A. L. Porous media: fluid transport and pore structure. Academic Press, 1991. https://doi.org/10.1016/B978-0-12-223651-8.50007-9

[9] Leontiev, N. E. Fundamentals of filtration theory: a tutorial. Moscow: Max Press, 2017.

[10] Whitaker, Stephen. "The Forchheimer equation: a theoretical development." Transport in Porous Media 25, no. 1 (1996): 27-61. https://doi.org/10.1007/BF00141261

[11] Ingham, Derek B., and loan Pop, eds. Transport phenomena in porous media III. Vol. 3. Elsevier, 2005.

[12] Tamer, O. S., E. S. Toropov, T. E. Shevnina, and T. I. Vorobieva. "Research of Reservoir Rock Properties in Violation of Darcy's Linear Law." In IOP Conference Series: Materials Science and Engineering, vol. 154, no. 1, p. 012006. IOP Publishing, 2016. https://doi.org/10.1088/1757-899X/154/1/012006

[13] Hassanizadeh, S. Majid, and William G. Gray. "High velocity flow in porous media." Transport in Porous Media 2, no. 6 (1987): 521-531. https://doi.org/10.1007/BF00192152

[14] Skjetne, Erik, and Jean-Louis Auriault. "High-velocity laminar and turbulent flow in porous media." Transport in Porous Media 36, no. 2 (1999): 131-147. https://doi.org/10.1023/A:1006582211517

[15] Bear, J. Dynamics of fluids in porous media. Elsevier, New York, 1972.

[16] Ewis, Karem Mahmoud. "Analytical Solution of Modified Bingham Fluid Flow through Parallel Plates Channel Subjected to Forchheimer Medium and Hall Current Using Linearized Differential Transformation Method." Journal of Advanced Research in Numerical Heat Transfer 4, no. 1 (2021): 14-31.

[17] Sukamta, Sukamta. "Computational Fluid Dynamics (CFD) and Experimental study of Two-Phase Flow Patterns GasLiquid with Low Viscosity in a Horizontal Capillary Pipe." CFD Letters 11, no. 8 (2019): 16-23.

[18] Sahak, Ahmad Sofianuddin A., Nor Azwadi Che Sidik, Siti Nurul Akmal Yusof, and Mahmoud Ahmed Alamir. "Numerical Study of Particle Behaviour in a Mixed Convection Channel Flow with Cavity using Cubic Interpolation Pseudo-Particle Navier-Stokes Formulation Method." Journal of Advanced Research in Numerical Heat Transfer 1, no. 1 (2020): 32-51.

[19] Nagasasikala, Madduleti, and Bommanna Lavanya. "Heat and mass transfer of a MHD flow of a nanofluid through a porous medium in an annular, circular region with outer cylinder maintained at constant heat flux." CFD Letters 11, no. 9 (2019): 32-58.

[20] Alexeev, B. V. "Analytic solution of nonlinear Leybenson equation in the theory of filtration." Fine Chemical Technologies 11, no. 1 (2016): 34-39. https://doi.org/10.32362/2410-6593-2016-11-34-39

[21] Abenov, M. M. "Chetirehmernaya matematika." Metody i prilozheniya. Nauchnaya monographia [Four-dimensional mathematics: Methods and applications. Scientific monograph](Almaty: Publishing House, 2019, 176 pp.).

[22] Abenov, M. M., M. B. Gabbasov, and F. Y. Ismagulova. "Movement of fluid inside the sphere." International Journal of Engineering \& Technology 7, no. 4 (2018): 42-44. https://doi.org/10.14419/ijet.v7i4.30.22001

[23] Abenov M. M., M. B. Gabbassov. Anyzotropnie chetirehmernie prostranstva ili novie kvaternioni [Anisotropic fourdimensional spaces or new quaternions] (Preprint, Nur-Sultan, 2020).

[24] Rakhymova, A. T., M. B. Gabbassov, and K. M. Shapen. "On one space of four-dimensional numbers." Journal of Mathematics, Mechanics and Computer Science 108, no. 4 (2020): 81-98. https://doi.org/10.26577/JMMCS.2020.v108.i4.07 